\title{
ВИКАРНЫЕ ВОСПОМИНАНИЯ: ОДИНАКОВО ЛИ ОНИ РАБОТАЮТ У НОСИТЕЛЕЙ РАЗЛИЧНЫХ КУЛЬТУРНЫХ МЕНТАЛИТЕТОВ? ${ }^{12}$
}

Нуркова В.В. (Московский государственный университет имени М.В.Ломоносова, Москва, Россия)

nourkova@mail.ru

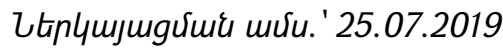

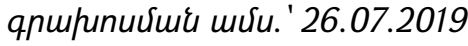

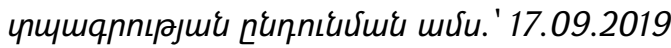

\begin{abstract}
Викарные воспоминания - это воспоминания о событиях, произошедших с другими людьми, которые, как показано, являются существенным компонентом личных историй. Целью данного исследования стала проверка гипотезы о том, что викарные воспоминания в большей степени свойственны представителям коллективистических, чем индивидуалистических культур. Мы также предположили, что функции викарных воспоминаний специфичны для различных культурных контекстов. 323 респондента-добровольца китайской $(\mathrm{N}=89)$, русской $(\mathrm{N}=142)$ и узбекской $(\mathrm{N}=92)$ этнической принадлежности продуцировали изображение последовательности событий своего детства на линии времени с их датировкой и графическим выражением эмоциональной насыщенности. В целом, женщины включали в изображение больше викарных воспоминаний, чем мужчины, а китайские респонденты - больше, чем русские и узбекские. Узбекские мужчины показали минимальную викарность. Анализ поддержал гипотезу о культурной функциональной специфике викарных воспоминаний. У китайских респондентов сочетание викарных воспоминаний и воспоминаний о личных достижениях направлено на реализацию директивной функции автобиографической памяти. На фоне универсальности данной функции, у русских респондентов викарные воспоминания связаны так же с саморефлексивной фрункцией, а у узбекских - с функцией эмоциональной саморегуляции.
\end{abstract}

Ключевые слова: автобиографическая память, викарные воспоминания, коллективизм/индивидуализм, Линия жизни, кросс-культурные исследования

Методология кросс-культурных исследований в психологии традиционно строится на сопоставлении частотных распределений выраженности того или иного психического френомена в сравниваемых выборках [8]. В исследованиях, проведенных в данной логике относительно автобиографической памяти способности преобразовывать эмпирику жизненного опыта в историю своей жизни [2], получены данные о количественных различиях между представителями т.н.

12 Исследование выполнено при финансовой поддержке РФФИ (проект №19-013-00387). 
индивидуалистических и коллективистических культур [9]. Так, первые устойчиво воспроизводят больший объем воспоминаний, начинают историю жизни с более раннего возраста, концентрируются на уникальных событиях прошлого и др. Однако не менее важной нам представляется линия исследований, связанная с предположением о том, что за фенотипически сходными явлениями в различных культурах может стоять качественно различный функционал. Данный факт может отчасти рефрлексироваться самими носителями культуры. Например, согласно самоотчётам, представители культур Китая и Японии по сравнению с североамериканцами чаще намеренно вспоминают события личного прошлого с целью извлечения жизненных уроков [4], в то время как тайцы обращаются к прошлому для его повторного эмоционально насыщенного «перепроживания» [5]. О функциональной специфике определенных фрорм автобиографических воспоминаний (порой ускользающей от рефлексии респондентов) могут свидетельствовать и различия в комплексах корреляционных связей с другими переменными. А.Зацепин [1] показал, что у москвичей субъективно яркие и детальные воспоминания о позитивных эпизодах прошлого связаны с доминированием ценностей поиска новых ощущений и гиперстимуляции, а у ташкентцев - с ценностями заботы о природе и других людях. Иными словами, в первом случае воспоминания развлекают, а во втором - конструируют групповую сопричастность.

В данном исследовании мы обратились к нетривиальному типу автобиографических воспоминаний - викарным воспоминаниям, где протагонистом является не сам вспоминающий, а другие люди [7]. Субъективное шкалирование показало, что викарные воспоминания не уступают по своим феноменологическим качествам и личностной значимости центрированным на себе воспоминаниям и часто спонтанно включаются в автобиографическую продукцию [6].

Следуя описанной выше логике фрункциональной специфичности, мы предположили, что качественные различия в функционировании викарных воспоминаний обнаружатся между представителями культур со сравнительно высоким (Россия - 39 баллов из 100) или низким (Китай - 20 баллов, Узбекистан - 22 балла) уровнем индивидуализма в сочетании с высоким (Китай - 66 баллов), средним (Узбекистан 50 баллов) или низким (Россия - 36 баллов) уровнем маскулинности (данные по https://www.hofstede-insights.com/product/comparecountries/). Согласно нашей гипотезе, в автобиографической памяти респондентов, принадлежащих к культуре с выраженным вектором маскулинности (Китай), викарные воспоминания преимущественно играют роль отражения конкурентного Другого, в соотнесении с которым строится индивидуальная самооценка, и поэтому они должны образовывать функциональный альянс с манифестирующими мотивацию достижения воспоминаниями в значимых для человека областях. В отличие от этого, в более фреминных культурах (Россия) фригура Другого выступает скорее в роли интериоризованного партнера по интимно-личностному общению и, таким образом, 
служит инстанцией внутреннего рефлексивного диалога. Наконец, коллективистическая доминанта культуры (Узбекистан) за счет размытости границы между «я» и «мы» предположительно может вовлекать викарные воспоминания в статусе орудия эмоциональной регуляции, так, что пережитые другим человеком события способны виртуально заменять собственный опыт.

Метод: В исследовании приняли участие 323 респондента-добровольца, из них 89 атрибутировали себя как носителей китайской культуры (М возраст $=30, \sigma=$ 8.99, 55\% женщин), 142 - русской культуры (М возраст $=32, \sigma=11.55,61 \%$ женщин) и 92 - узбекской культуры (М возраст = 30, $\sigma=11.88,56 \%$ женщин). Для экспликации макроструктуры автобиографической памяти применялась методика «Линия детства», при выполнении которой респонденты продуцировали на листе формата А4 изображение последовательности наиболее запомнившихся событий своего детства, датируя и кратко называя каждое из воспоминаний, соотнося его с временной осью и выражая его эмоциональную насыщенность расстоянием от оси (позитивные в верхней полуплоскости, негативные - в нижней полуплоскости). Память о периоде детства была выбрана для анализа в связи с тем, что воспоминания о данном периоде жизни высоко селективны и преимущественно портретируют устойчивые личностные характеристики респондентов, в отличие от воспоминаний о недавнем прошлом, содержание которых ситуативно варьирует [3].

Воспоминания викарного, “достиженческого» (как позитивный, так и негативный результат собственной активности в состязательном контексте) и «группового» (совместные действия с другими людьми) типов выделялись на основе экспертных оценок (тремя независимыми экспертами), уровень согласованности оценок достигал 95\%.

Результаты:В совокупности участники исследования актуализировали 2826 воспоминаний о детстве. Представители российской выборки продуцировали максимальное количество воспоминаний $(M=11.36, \sigma=6.13)$, демонстрируя значимо более высокую продуктивность $(F(2,323)=42.941, p<.001$ после корректировки для множественных сравнений Бонферрони, $\left.\eta^{2}=.336\right)$, чем представители китайской $(M=6.18, \sigma=2.12)$ и узбекской $(M=7.09, \sigma=3.39)$ выборок. 396 воспоминаний были отнесены экспертами к категории викарных, например, “родители развелись» (китаянка), «в деревне родился брат» (китаянка), «учитель постоянно забывал имя одного из учеников в классе» (китаец), «мой двоюродный брат женился» (русский), «мой брат победил на соревнованиях по фрутболу» (русская), «моя мама сшила себе красивое красное платье» (узбечка), «соседскому мальчику родители подарили игрушечный автомобиль» (узбек), и т.п.

Двухфакторный дисперсионный анализ 2 (пол: мужской, женский) х 3 (этничность: китайцы, русские, узбеки) для количества викарных воспоминаний в изображении детства в качестве зависимой переменной показал наличие главного эффректа пола $\left(\mathrm{F}(1,324)=11.081, \mathrm{p}=.001, \eta^{2}=.034\right)$, массированный эфрфект 
этнической принадлежности $\left(F(2,323)=34.944, p<.001, \eta^{2}=.180\right)$ и отсутствие взаимодействия между факторами $(F(1,321)=0.270, p=.764)$. В целом, китайские респонденты значительно превосходили остальных участников исследования в количестве викарных воспоминаний при отсутствии внутригрупповых гендерных различий (мужчины $M=1.88, \sigma=1.28$; женщины $M=2.196, \sigma=1.36, p<.001$ после корректировки для множественных сравнений Бонферрони), в то время как русские женщины $(M=1.28 \sigma=1.22)$ и узбечки $(M=0.88, \sigma=1.23)$ включали в Линию детства больше викарных воспоминаний, чем мужчины из соответствующих групп (русские $-M=0.741, \sigma=1.04$; узбеки $-M=0.38, \sigma=0.60$ ). Несмотря на то, что китайские респонденты, как было показано выше, в целом генерировали менее событийно насыщенные «Линии детства», аналогичные результаты дисперсионного анализа были получены и для процентной представленности викарных воспоминаний (эффрект пола $p=.04$; эффрект этнической принадлежности $p<.001$ ): китайские мужчины - $M=33.53 \%, \sigma=22.44$; китайские женщины $-M=34.50 \%, \sigma=21.31$; русские мужчины - $M=7.24 \%, \sigma=26$; русские женщины - $M=11.19 \%, \sigma=11.73$; узбекские мужчины - $M=5.83 \%, \sigma=9.76$; узбекские женщины $-M=11.87 \%, \sigma=$ 15.09. Таким образом, мы видим, что принадлежность к коллективистическому типу культуры сама по себе не является надежным предиктором викарности автобиографрической памяти, так как представители высоко коллективистических культур могут демонстрировать как высокую (Китай), так и низкую (Узбекистан) выраженность данного показателя.

Корреляционный анализ подтвердил, что во всех трех выборках количество викарных воспоминаний значимо положительно взаимосвязано с количеством «достиженческих" воспоминаний (китайцы - $r=.542, p<.001$; русские $-r=.274, p$ $=.001 ;$ узбеки $-r=.319, p=.002)$. Однако регрессионный анализ обнаружил, что коэффициент детерминации количества викарных воспоминаний количеством "достиженческих" воспоминаний существенно различается между группами. Так для группы китайских респондентов регрессионное уравнение имеет вид: Количество викарных воспоминаний = 0.776 х количество "достиженческих" воспоминаний + $1.295+/-1.12$, объясняя максимальные по трем выборкам $29.3 \%$ общей дисперсии $\left(\mathrm{R}^{2}=.293, \mathrm{~F}(1,88)=36.116, \beta=0.542, \mathrm{~b}=0.776\right.$, e = 1.12, const $\mathrm{b}=$ $1.295, p<.001)$. Аналогично для узбекской группы, хотя и с менее убедительными параметрами модели $\left(\mathrm{R}^{2}=.102, \mathrm{~F}(1,91)=10.1992, \beta=0.199, \mathrm{~b}=0.634\right.$, e = 1.02, const $b=0.523, p=.002)$, число «достиженческих» воспоминаний объясняет $10.2 \%$ дисперсии. В то же время для группы русских респондентов коэффрициент детерминации равен .074 , т.е. объясняет всего $7.4 \%$ дисперсии $\left(\mathrm{R}^{2}=.074, \mathrm{~F}(1,141)\right.$ $=11.316, \beta=0.118, b=0.398$, $e=1.13$, const $b=0.833, p=.001)$.

Таким образом, можно утверждать, что именно в китайской культуре с максимальным уровнем маскулинности, т.е. с доминированием ценностей соревнования и создания вертикальных социальных связей, в модели автобиогра- 
фрической памяти выявляется наиболее устойчивая функциональная ассоциация между "достиженческими» и викарными воспоминаниями. Суть данной сопряженности, по нашему мнению, заключается в репрезентации Другого либо как модели, с которой соотносятся личные достижения, либо как соперника в конкурентных ситуациях. В культуре с низким уровнем маскулинности (Россия), где более востребованы ценности поддержания эмоционального комфорта и горизонтальных социальных связей, данная взаимосвязь теряет свою прогностическую силу. Представители узбекской культуры предсказуемо заняли промежуточную позицию.

Затем нами был проведен регрессионный анализ с пошаговым исключением переменных для каждой из этнических групп с целью выявления культурно специфичных комплексов взаимосвязей. В качестве потенциальных предикторов количества викарных воспоминаний тестировался ряд переменных: возраст, общее количество воспоминаний, процент позитивных и негативных воспоминаний, индекс позитивности (значение >1 при преобладании положительных воспоминаний), возраста наиболее раннего и наиболее позднего воспоминаний, субъективная протяженность детства, количество и процент «групповых» и «достиженческих» воспоминаний.

Для китайской выборки значимыми предикторами количества викарных воспоминаний стали: количество «достиженческих" воспоминаний (b = 0.803), общее количество воспоминаний, нанесенных на «Линию детства» $(b=0.265)$ и, с отрицательным вкладом, количество воспоминаний о членстве в социальных группах (b =-0.285), при $\mathrm{R}^{2}=.508, \mathrm{~F}(3,85)=29.275, \mathrm{p}<.001, \mathrm{e}=0.943$, const $\mathrm{b}$ $=0.059$ (ns). Иными словами, как было описано выше, индивидуальная насыщенность автобиографической памяти викарными воспоминаниями предсказывается высоким количеством воспоминаний о личных достижениях и провалах, возрастает с ростом общего объема воспоминаний (как мы помним, в целом воспоминания членов этой группы относительно малочисленны) и, наоборот, снижается в присутствии ориентированных на социальную группу воспоминаний. Таким образом, достаточно парадоксально для коллективистического менталитета, в данном случае, осознавание себя частью группы препятствует викарности памяти.

Для русской выборки в качестве значимых предикторов количества викарных воспоминаний выступают лишь “достиженческие» воспоминания (b = 0.267) и общее количество воспоминаний $(\mathrm{b}=0.054)$ при $\mathrm{R}^{2}=.145, \mathrm{~F}(2,140)=11.860, \mathrm{p}<$ $.001, \mathrm{e}=1.09$, const $\mathrm{b}=0.294$ (ns). Поскольку основной вклад в процент объясняемой дисперсии делает именно общая продуктивность памяти, предположим, что викарные воспоминания русских респондентов представляют собой материал для рефлексии своей личности и жизненного пути и не образуют устойчивых связей с другими типами воспоминаний (кроме «достиженческих»).

Своеобразную картину мы наблюдаем у узбекской выборки. Здесь, кроме уже стандартных «достиженческих» воспоминаний $(b=0.488)$ и общей продуктивности 
(b = 0.124), предикторами викарности с обратным знаком становятся эмоциональный индекс картины детства $(\mathrm{b}=-0.125)$ и возраст последнего внесенного в изображение события $(\mathrm{b}=-0.065)$ при $\mathrm{R}^{2}=.280, \mathrm{~F}(4,87)=8.473, \mathrm{p}<.001$, e = 0.93 , const $b=0.940$. Таким образом, негативное эмоциональное отношение к опыту детства в совокупности с его ранним субъективным завершением провоцирует насыщение картины прошлого викарными воспоминаниями. Полученные результаты, по нашему мнению, позволяют предположить компенсаторный фрункционал викарных воспоминаний в данной группе. Включение в свою (короткую и не слишком счастливую) историю детства событий из жизни других людей, при условии свойственной коллективистическим культурам проницаемости межличностных границ, способно отчасти компенсировать неудовлетворенность и наполнить память о детстве вариативными эмоциями.

Выводы: Гипотеза о потенциальной функциональной кросс-культурной вариативности викарных воспоминаний (воспоминаний, протагонистом которых выступает не вспоминающий, а другой человек) была рассмотрена на материале китайской, русской и узбекской выборок. Универсальной кросс-культурно устойчивой функцией викарных воспоминаний следует признать создание репрезентаций мотивационно значимых социальных моделей для мониторинга самооценки (директивная функция), что эмпирически проявляется в корреляционном альянсе "достиженческих" и викарных воспоминаний. Данная взаимосвязь объясняет 29.3\%, 10.2\% и 7.4\% дисперсии количества викарных воспоминаний для китайской, узбекской и русской выборок соответственно. Однако дальнейший анализ показал, что корпус викарных воспоминаний может быть использован и для реализации иных функций, которые являются культурно уникальными. Так, в китайской выборке викарные воспоминания ассоциированы с сокращением числа «групповых» воспоминаний, что возможно делает их средством поддержания своеобразного психологического иммунитета против размывания личных границ в коллективистическом контексте. В узбекской выборке, наоборот, тенденция к психологическому слиянию с группой делает викарные воспоминания потенциальным орудием эмоциональной саморегуляции. Специфика русской выборки предполагает толкование Другого как интериоризованного субъекта внутреннего диалога, что предположительно позволяет викарным воспоминаниям играть роль инструмента рефрлексии своего жизненного пути.

\section{Литература}

1. Зацепин А.С. Функциональные особенности структурных единиц автобиографической памяти в различных культурах (на материале выборок из Москвы и Ташкента) // Дипломная работа, МГУ имени М.В.Ломоносова, 2016.

2. Нуркова В.В. Автобиографическая память С позиций культурнодеятельностной психологии: результаты и перспективы исследования // 
Вестник Московского университета. Серия 14: Психология, 2011, № 1, С. 7990.

3. Нуркова В. В. Самоопределяющие нарративы в развитии личности // Психологическая наука и образование, 2014, № 4, С. 22-30.

4. Alea, N., Wang, Q. Going Global: The Functions of Autobiographical Memory in Cultural Context // Memory, Special Issue, 2015, Vol. 23, №.1., pp. 1-10.

5. Liao, H. W., Bluck, S., Alea, N., Cheng, C. L. Functions of autobiographical memory in Taiwanese and American emerging adults // Memory, 2016, Vol. 24, №.4, pp. 423-436.

6. Lind, M., Thomsen, D. K. Functions of personal and vicarious life stories: identity and empathy //Memory, 2018, Vol. 26, №.5, pp. 672-682.

7. Pillemer, D. B., Steiner, K. L., Kuwabara, K. J., Thomsen, D. K., Svob, C. Vicarious memories// Consciousness and Cognition, 2015, №. 36, pp. 233-245.

8. Taras, V., Rowney, J., Steel, P. Half a century of measuring culture: Review of approaches, challenges, and limitations based on the analysis of 121 instruments for quantifying culture// Journal of International Management, 2009, Vol.15, №. 4, pp. 357-373.

9. Wang Q., Ross M. Culture and memory / Handbook of cultural psychology. Ed by S. Kitayama, D. Cohen. New York, NY: Guilford Press, 2007, pp. 645-667.

\section{VICARIOUS MEMORIES: DO THEY PERFORM THE SAME JOB IN DIFFERENT CULTURAL MINDS?}

Nourkova V.V. (Lomonosov Moscow State University, Moscow, Russia)

Vicarious memories are recollections of events that happened to other people. They were recently recognized as a meaningful component of personal life story. In this study we examined whether vicarious memories were more common in collectivistic than in individualistic cultures and speculated that they might serve different functions in different cultural contexts. 323 participants with Chinese $(\mathrm{N}=89)$, Russian $(\mathrm{N}=142)$, and Uzbek ( $\mathrm{N}=92)$ background drew a lifeline of their childhood. In general, females produced more vicarious lifelines than males and the Chinese participants produced more vicarious lifelines than their Russian and Uzbek female counterparts. The Uzbek male participants generated the least vicarious lifelines. However, the functionality of vicarious memories varies among cultures. In Chinese participants the conjunction of vicarious and achievement memories serves a directive function. Although the directive function affirmed to be the gist of vicarious memories, in Russian participants they are relevant also to a self-reflective function. Accordingly, in Uzbek participants they are associated with a function of emotional regulation.

Keywords: autobiographical memory, vicarious memories, collectivism/individualism, lifeline, cross-cultural studies. 